\title{
Recidivas de endometriomas ováricos tras cirugía laparoscópica
}

\author{
Nayara López C. 1, Lucía Paz R. ${ }^{1}$, Alicia Hernández G. 1, Javier De Santiago G. 1, \\ Belén San José $V$. ${ }^{a}$ \\ 1 Servicio de Ginecología, Hospital Universitario La Paz, Madrid, España. \\ a Bioestadística.
}

\section{RESUMEN}

Objetivo: Analizar las variables que influyen en la recurrencia tras la extirpación laparoscópica de endometriomas ováricos. Métodos: Se estudiaron retrospectivamente 214 pacientes, intervenidas por laparoscopia entre los años 2005 y 2006 en el Hospital La Paz y seguidas por 5 años. Como criterios de recurrencia se consideraron: dolor, hallazgo ecográfico y elevación de Ca 125. Resultados: El 30,8\% (66/214) presentaron recurrencia del dolor, el 28\% (60/214) recidiva ecográfica y el 16,8\% (36/214) Ca 125 elevado. Las pacientes con recidiva sintomática presentaron mayor dismenorrea y dispareunia previas a la cirugía $(6,8 \pm 2,5$ y $1,3 \pm 2,5$ frente a $3,8 \pm 3,4$ y $0,2 \pm 1,0$ en las no recurrencias $(p=0,0001 ; p=0,0001)$. La puntuación de dismenorrea y dispareunia previas también fue superior en recidiva ecográfica $(5,9 \pm 3,3$ y 1,2 $\pm 2,5$ frente a 4,3 $\pm 3,4$ y $0,2 \pm 1,1$ en ausencia de recidiva) ( $p=0,003 ; p=0,002)$. La recurrencia de dismenorrea fue mayor en jóvenes $(31,3 \pm 5,4$ años versus $34,3 \pm 7,8 ; p=0,02$ ), con quistectomía ( $35 \%$ versus $16,7 \%$ en anexectomía; $\mathrm{p}=0,02)$ y adherensiolisis $(46,4 \%$ versus $23,4 \% ; \mathrm{p}=0,001)$ y con tratamiento médico posquirúrgico $(41,5 \%$ frente 22,5\%; $p=0,004)$. El tiempo medio de no recurrencia sintomática fue 44 meses (IC95\%: 41-47) y el de no recidiva ecográfica de 47 meses (IC95\%:45-50). Conclusión: El grado de dismenorrea y dispareunia previas a la cirugía fue el factor más claramente asociado a la recidiva.

\section{PALABRAS CLAVE: Recurrencia, dolor, endometrioma, laparoscopia}

\section{SUMMARY}

Objective: To analyze the variables influencing recurrence after laparoscopic excision of ovarian endometrioma. Methods: We retrospectively studied 214 cases who underwent laparoscopic surgery between 2005 and 2006 in the Hospital La Paz and followed for 5 years. As recurrence criteria were considered: pain, ultrasound finding and Ca125 elevation. Results: $30.8 \%$ (66/214) of patients presented pain recurrence, $28 \%$ (60/214) ultrasound recurrence and 16.8\% (36/214) Ca 125 elevation. Patients with symptomatic recurrence had a bigger degree of dysmenorrhea and dyspareunia before surgery $(6.8 \pm 2.5$ and $1.3 \pm 2.5$ against 3.8 \pm 3.4 and $0.2 \pm 1.0$ in no recurrence ones $(p=0.0001 ; p=0.0001)$. Previous dysmenorrheal and dyspareunia punctuation was also greater in ultrasound recurrences $(5.9 \pm 3.3$ and $1.2 \pm 2.5$ against $4.3 \pm 3.4$ and 0.2 \pm 1.1 of those with no recurrence) $(p=0.003 ; p=0.002)$. Dysmenorrhea recurrence was greater in young $(31.3 \pm 5.4$ years old versus $34.3 \pm 7.8 ; p=0.02)$, with cystectomy $(35 \%$ versus $16.7 \%$ in adnexectomy; $\mathrm{p}=0.02)$, adhesiolysis $(46.4 \%$ versus $23.4 \% ; \mathrm{p}=0.001)$ and with medical treatment after surgery $(41.5 \%$ against 22.5\%; $p=0.004)$. The mean time of no symptomatic recurrence was 44 months (Cl95\%:41-47) and the no ultrasound recurrence was 47 months (Cl95\%:45-50). Conclusion: The degree of dysmenorrhea and dyspareunia before surgery was the factor most clearly associated with recurrence. 


\section{INTRODUCCIÓN}

La endometriosis afecta al $5-12 \%$ de mujeres en edad reproductiva, llegando hasta el $50 \%$ en infértiles $(1,2)$. La cirugía es la terapia más adecuada en la endometriosis ovárica ya que el tratamiento médico aislado resulta insuficiente $(3,4)$. La laparoscopia es la técnica de elección por su baja morbilidad, alta tolerancia y bajo coste y en relación a la mejora de los síntomas (5). Uno de los puntos más frustrantes es la alta tasa de recurrencia $y$, actualmente, pocos factores de riesgo han sido consistentemente identificados. La recurrencia ecográfica oscila entre un $8-16 \%$ a los $2-3$ años llegando al $32 \%$ a los 5 años, mientras que la recidiva sintomática se aprecia en el $10-76 \%$ de las pacientes $(1,2,6)$.

El objetivo de este estudio es analizar los factores implicados en la recurrencia tras la extirpación laparoscópica de endometriomas ováricos. Y como objetivo secundario, evaluar las características, evolución y tratamiento de las recidivas.

\section{PACIENTES Y MÉTODO}

Se estudiaron retrospectivamente 214 pacientes con endometrioma ovárico intervenidas por laparoscopia entre los años 2005 y 2006, en el Hospital Universitario La Paz, Madrid, España. El ovario fue la única localización de la endometriosis. Se efectuó un seguimiento durante 5 años, con revisión a los 3 meses poscirugía, luego semestral los 2 primeros años y anual posteriormente. Fueron consideradas infértiles las pacientes con deseo genésico y búsqueda infructuosa de gestación de 6 meses a 1 año.

Como características previas a la cirugía, se evaluaron: edad, síntomas (dismenorrea [escala de 0-10], dispareunia [escala de 0-10], infertilidad), Ca-125 $(\mathrm{mUl} / \mathrm{ml})$, hallazgo ecográfico de miomas o adenomiosis, número de quistes, tamaño y lateralidad. Se estudió la administración de tratamiento médico pre y pos quirúrgico, tipo de intervención (quistectomía, anexectomía), cirugía realizada por médico adjunto o residente como primer cirujano, realización de adherensiolisis y la asociación a rotura del quiste e implantes peritoneales superficiales. También se analizaron las características de la recidiva, su evolución y tratamiento.

Se consideró como hallazgo ecográfico sugestivo de endometrioma a la formación ovárica predominantemente quística $>1,5 \mathrm{~cm}$, con pared de 1-4 $\mathrm{mm}$ y contenido principalmente sonoluscente, pero con ecos en su interior de baja ecogenicidad y distribución más o menos homogénea. El Ca 125 se consideró alto por encima de $35 \mathrm{Ul} / \mathrm{ml}$, límite establecido por el laboratorio del hospital.
La recurrencia fue definida en tres términos: reaparición de dismenorrea, hallazgo ecográfico persistente sugestivo de endometrioma o nueva elevación de $\mathrm{Ca} 125$ en los casos que previamente estaban altos. La indicación de la segunda cirugía estuvo basada en el tamaño del quiste $(>4 \mathrm{~cm})$ y en sintomatología intensa.

Los datos se procesaron mediante el programa estadístico SPSS versión 11. Para el estudio descriptivo se utilizó la media con la desviación estándar para variables cuantitativas continuas y frecuencias absolutas y relativas expresadas en porcentaje para las cualitativas. Las comparaciones entre variables cuantitativas continuas se realizaron mediante pruebas no paramétricas (Kruskal-Wallis, U- Mann-Whitney) y entre cualitativas mediante la prueba de la $x^{2}$ o el test exacto de Fisher. El tiempo de no recurrencia se evaluó mediante curvas de Kaplan-Meyer y de Hazard. El valor $p<0,05$ indicó diferencia estadísticamente significativa.

\section{RESULTADOS}

Las características de las pacientes previas a la cirugía se detallan en la Tabla I. Recibieron tratamiento médico previo a la intervención el 10,7\% de las pacientes (23/214). De ellas, el 91,3\%(21/23) recibió anticonceptivos orales y el 8,7\% (2/23) análogos de GnRh. El tiempo medio de tratamiento fue de $17,5 \pm 33,5$ meses.

El $43,9 \%(94 / 214)$ recibieron tratamiento poscirugía. El 56,4\% (53/94) recibió anticonceptivos, $31,9 \%$ (30/94) análogos de GnRh, 8,5\%(8/94) la combinación de ambos y 3,2\% (3/94) DIU con levonorgestrel. El tiempo medio de tratamiento fue 17,8 $\pm 16,2$ meses. Consiguen una gestación posquirúrgica el $14,5 \%(31 / 214)$ de las pacientes.

El $41,5 \%$ (89/214) de los casos presentaron alguna forma de recurrencia. Las distintas opciones de recidiva se detallan en la Figura 1.

Hallazgos quirúrgicos: Se realizó cirugía conservadora, quistectomía, en el 77,6\% (166/214) de los casos y en el $22,4 \%(48 / 214)$ restante, anexectomía. La cirugía fue efectuada por un médico adjunto en el $65,9 \%(141 / 214)$ de los casos y por un residente en el 34,1\% (73/214). En el 5,1\% (11/214) de las pacientes, el endometrioma estaba roto en el momento de la cirugía. Existían adherencias relevantes procediéndose a la adherensiolisis en el $32,2 \%(69 / 214)$ de los casos. Se hallaron implantes peritoneales superficiales en el 18,2\% (39/214). La relación de los diversos factores con las distintas formas de recurrencia se evalúa en las Tablas II-V.

Características de las recurrencias: En 34/60 casos la recurrencia ecográfica $(56,7 \%)$ fue ipsilateral, 
Tabla I

CARACTERÍSTICAS PREVIAS A LA CIRUGÍA

Edad media (años)

Dismenorrea

Dispareunia

Disquecia

Trastornos miccionales

Infertilidad

Ca $125>35 \mathrm{mUl} / \mathrm{ml}$

Ca $125 \mathrm{mUl} / \mathrm{ml}$

Miomas

Adenomiosis

1 quiste

2 quistes

Quiste izquierdo

Quiste derecho

Quistes bilaterales

Ningún hijo previo

1 hijo previo

Tamaño medio ecográfico 1er quiste

Tamaño medio ecográfico $2^{\circ}$ quiste

Tamaño medio laparoscópico 1er quiste

Tamaño medio laparoscópico $2^{\circ}$ quiste

\author{
$33,4 \pm 7,3 ; p 50=32,0$ \\ $74,8 \%(160 / 214)$ \\ $11,2 \%(24 / 214)$ \\ $2,8 \%(6 / 214)$ \\ $0,5 \%(1 / 214)$ \\ $21 \%(45 / 214)$ \\ $64,4 \%(116 / 183)$ \\ $66,1 \pm 71,5 ;$ p $50=49,4$ \\ $22,4 \%(48 / 214)$ \\ $4,2 \%(9 / 214)$ \\ $74,7 \%(160 / 214)$ \\ $25,2 \%(54 / 214)$ \\ $58,4 \%(97 / 166)$ \\ $41,6 \%(69 / 166)$ \\ $22,4 \%(48 / 214)$ \\ $69,1 \%(148 / 214)$ \\ $59,1 \%(39 / 66)$ \\ $48,0 \pm 17,8 \mathrm{~mm} ; \mathrm{p} 50=45,5 \mathrm{~mm}$ \\ $31,4 \pm 13,6 \mathrm{~mm} ; \mathrm{p} 50=27,5 \mathrm{~mm}$ \\ $48,7 \pm 20,3 \mathrm{~mm} ; \mathrm{p} 50=44,0 \mathrm{~mm}$ \\ $34,4 \pm 19,7 \mathrm{~mm} ; \mathrm{p} 50=30,0 \mathrm{~mm}$
}

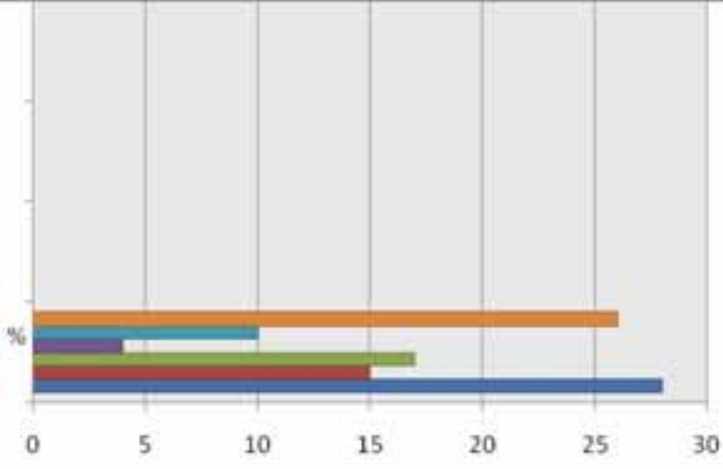

DDISMENORREA+ECOGRAFICO+CA

125ALTO

EECOGRAFICO+CA125ALTO

- DISMENORREA+CA125ALTO

DISMENORREA+ECOGRAFICA

- ECOGRAFICA

- DISMENORREA.

Figura 1. Distintas posibilidades de recurrencia.

en $14 / 60(23,3 \%)$ contralateral, en $9 / 60(15 \%)$ bilateral y en el $5 \%(3 / 60)$ restante en el trayecto de algún trocar. El tamaño medio del quiste fue de 43,6 $\pm 25,9$ $\mathrm{mm}$.

De un total de 89 pacientes, $23(25,8 \%)$ recibie- ron tratamiento hormonal como terapia de estabilización de la recidiva, en $43(48,4 \%)$ el tratamiento con AINEs fue suficiente para control del dolor y en 23 pacientes $(25,8 \%)$ se recurrió a nueva cirugía. El $92,1 \%(82 / 89)$ de las recidivas se mantuvieron estables con el primer tratamiento (médico/quirúrgico) llegándose a requerir una tercera cirugía en el 7,9\% (7/89) de los casos.

El tiempo medio de no recurrencia sintomática fue 44 meses (IC95\%: 41-47 meses), el de no recidiva ecográfica de 47 meses (IC95\%: 45-50 meses) y el de no elevación de Ca125 posquirúrgico de 52 meses (IC95\%: 50-55 meses) (Figura 2).

Subdividiendo los tipos de recurrencia en tres periodos ( $\leq 6$ meses, 7-12 meses, $>12$ meses), se observó que el mayor grupo de recidiva sintomática fue en $\leq 6$ meses $(51,5 \%)$, de recurrencia ecográfica entre los $7-12$ meses $(44,1 \%)$ y de nueva elevación del Ca 125 de 7-12 meses (47,2\%). En los 3 grupos de recurrencia, el porcentaje menor de recidiva fue $>12$ meses (12,1\% para síntomas, 35,6\% para ecografía y 30,6 \% para elevación de Ca 125).

\section{DISCUSIÓN}

Existe gran diversidad de criterios para definir la recurrencia. Hay estudios que sólo consideran la 
Tabla II

RECURRENCIA DE SÍNTOMAS

\begin{tabular}{|c|c|c|c|}
\hline & $\begin{array}{l}\text { Recurrencia } \\
\text { síntomas }\end{array}$ & $\begin{array}{l}\text { No recurrencia } \\
\text { síntomas }\end{array}$ & Valor $\mathrm{p}<$ \\
\hline Edad (años) & $31,3 \pm 5,4$ & $34,3 \pm 7,8$ & 0,02 \\
\hline Dismenorrea $(0 / 10)$ & $6,8 \pm 2,5$ & $3,8 \pm 3,4$ & 0,0001 \\
\hline Dispareunia $(0 / 10)$ & $1,3 \pm 2,5$ & $0,2 \pm 1,0$ & 0,0001 \\
\hline Infertilidad $n=45$ & $18(40 \%)$ & $27(60 \%)$ & NS \\
\hline Ca 125 alto $(\mathrm{mUl} / \mathrm{ml})$ & $71,1 \pm 67,4$ & $61,0 \pm 72,8$ & NS \\
\hline Miomas $n=48$ & $10(20,8 \%)$ & $38(79,2 \%)$ & NS \\
\hline Adenomiosis $n=9$ & $5(55,6 \%)$ & $4(44,4 \%)$ & NS \\
\hline Número de quistes & $1,2 \pm 0.5$ & $1,2 \pm 0.4$ & NS \\
\hline Unilateral $n=166$ & $50(30,1 \%)$ & $116(69,9 \%)$ & NS \\
\hline Bilateral $n=48$ & $16(33,3 \%)$ & $32(66,7 \%)$ & NS \\
\hline Derecho $n=69$ & $19(27,5 \%)$ & $50(72,5 \%)$ & NS \\
\hline Izquierdo $\mathrm{n}=97$ & 35 (36\%) & $62(64 \%)$ & NS \\
\hline Número de gestaciones previas viables & $0,2 \pm 0,5$ & $0,5 \pm 0,8$ & 0,02 \\
\hline Tamaño 1er quiste laparoscopia (mm) & $50,5 \pm 23,4$ & $47,7 \pm 18,6$ & NS \\
\hline Tamaño $2^{\circ}$ quiste laparoscopia $(\mathrm{mm})$ & $41,0 \pm 25,6$ & $31,4 \pm 16,1$ & NS \\
\hline Tratamiento médico precirugía $n=23$ & $5(21,7 \%)$ & $18(78,3 \%)$ & NS \\
\hline Con tratamiento médico poscirugía $n=94$ & $39(41,5 \%)$ & $55(58,5 \%)$ & 0,004 \\
\hline Sin tratamiento médico poscirugía $n=120$ & $27(22,5 \%)$ & $93(77,5 \%)$ & \\
\hline
\end{tabular}

NS: diferencia no significativa.

Tabla III

RECURRENCIA ECOGRÁFICA

\begin{tabular}{|c|c|c|c|}
\hline & $\begin{array}{r}\text { Recurrencia } \\
\text { ecográfica }\end{array}$ & $\begin{array}{l}\text { No recurrencia } \\
\text { ecográfica }\end{array}$ & Valor $\mathrm{p}<$ \\
\hline Edad (años) & $31,9 \pm 5,7$ & $33,9 \pm 7,7$ & NS \\
\hline Dismenorrea $(0 / 10)$ & $5,9 \pm 3,3$ & $4,3 \pm 3,4$ & 0,003 \\
\hline Dispareunia $(0 / 10)$ & $1,2 \pm 2,5$ & $0,2 \pm 1,1$ & 0,002 \\
\hline Infertilidad $n=45$ & $19(42,2 \%)$ & $26(57,8 \%)$ & 0,02 \\
\hline Ca 125 alto (mUl/ml) & $72,7 \pm 67,5$ & $61,5 \pm 72,6$ & 0,05 \\
\hline Miomas $\mathrm{n}=48$ & $11(23 \%)$ & $37(77 \%)$ & NS \\
\hline Adenomiosis $n=9$ & $5(55,6 \%)$ & $4(44,4 \%)$ & NS \\
\hline Número quistes & $1,3 \pm 0,4$ & $1,2 \pm 0,4$ & NS \\
\hline Unilateral $n=166$ & $43(26 \%)$ & $123(74 \%)$ & NS \\
\hline Bilateral $n=48$ & $17(35,4 \%)$ & $31(64,6 \%)$ & \\
\hline Derecho $\mathrm{n}=69$ & $17(24,6 \%)$ & $52(75,4 \%)$ & NS \\
\hline Izquierdo $n=97$ & $27(27,8 \%)$ & $70(72,2 \%)$ & \\
\hline Número de gestaciones previas viables & $0,4 \pm 0,8$ & $0,5 \pm 0,7$ & NS \\
\hline Tamaño 1er quiste laparoscópico & $50,1 \pm 21,9$ & $48,1 \pm 19,6$ & NS \\
\hline Tamaño $2^{\circ}$ quiste laparoscópico & $38,3 \pm 25,8$ & $33,0 \pm 16,6$ & NS \\
\hline Tratamiento médico precirugía $n=23$ & $5(21,7 \%)$ & $18(78,3 \%)$ & NS \\
\hline Tratamiento médico poscirugía $n=94$ & $29(30,8 \%)$ & $65(69,2 \%)$ & NS \\
\hline
\end{tabular}

NS: diferencia no significativa. 
Tabla IV

RECURRENCIA NUEVA ELEVACIÓN DEL Ca 125

\begin{tabular}{|c|c|c|c|}
\hline & $\begin{array}{l}\text { Recurrencia } \\
\text { Ca } 125\end{array}$ & $\begin{array}{l}\text { No recurrencia } \\
\quad \mathrm{Ca} 125\end{array}$ & Valor $\mathrm{p}<$ \\
\hline Edad (años) & $31,5 \pm 5,8$ & $33,7 \pm 7,5$ & NS \\
\hline Dismenorrea $(0 / 10)$ & $5,4 \pm 3,3$ & $4,6 \pm 3,4$ & NS \\
\hline Dispareunia $(0 / 10)$ & $1,1 \pm 2,3$ & $0,4 \pm 1,5$ & 0,02 \\
\hline Infertilidad $n=45$ & $10(22,2 \%)$ & $35(77,8 \%)$ & NS \\
\hline Ca 125 alto (mUl/ml) & $77,4 \pm 71,3$ & $62,1 \pm 71,1$ & 0,01 \\
\hline Miomas $n=48$ & $7(14,6 \%)$ & $41(85,4 \%)$ & NS \\
\hline Adenomiosis $n=9$ & $2(22,2 \%)$ & $7(77,8 \%)$ & NS \\
\hline Número quistes & $1,2 \pm 0.4$ & $1,2 \pm 0,4$ & NS \\
\hline Unilateral $n=166$ & $27(16,3 \%)$ & $139(83,7 \%)$ & NS \\
\hline Bilateral $n=48$ & $9(18,8 \%)$ & $39(81,2 \%)$ & \\
\hline Derecho $n=69$ & $8(11,6 \%)$ & $61(88,4 \%)$ & NS \\
\hline Izquierdo n=97 & $20(20,6 \%)$ & $77(79,4 \%)$ & \\
\hline Número de gestaciones previas viables & $0,2 \pm 0,5$ & $0,5 \pm 0,8$ & NS \\
\hline Tamaño laparoscópico 1er quiste & $51,7 \pm 24,6$ & $48,0 \pm 19,2$ & NS \\
\hline Tamaño laparoscópico $2^{\circ}$ quiste & $48,3 \pm 37,1$ & $32,7 \pm 15,8$ & NS \\
\hline Tratamiento médico precirugía $n=23$ & $3(13 \%)$ & $20(87 \%)$ & NS \\
\hline Tratamiento médico poscirugía $\mathrm{n}=94$ & $18(19,1 \%)$ & $76(80,9 \%)$ & NS \\
\hline
\end{tabular}

NS: diferencia no significativa.

Tabla V

CARACTERÍSTICAS DE LA CIRUGÍA

\begin{tabular}{|c|c|c|c|c|c|c|}
\hline Cirugía & $\begin{array}{r}\text { Recurrencia } \\
\text { síntomas }\end{array}$ & $\begin{array}{l}\text { Valor } \\
\mathrm{p}<\end{array}$ & $\begin{array}{l}\text { Recurrencia } \\
\text { ecográfica }\end{array}$ & $\begin{array}{l}\text { Valor } \\
\mathrm{p}<\end{array}$ & $\begin{array}{l}\text { Recurrencia } \\
\text { Ca } 125\end{array}$ & $\begin{array}{c}\text { Valor } \\
\mathrm{p}<\end{array}$ \\
\hline Quistectomía n=166 & $58(35 \%)$ & 0,02 & $50(30,2 \%)$ & NS & $32(19,3 \%)$ & NS \\
\hline Anexectomía $n=48$ & $8(16,7 \%)$ & & $10(20,8 \%)$ & & $4(8,3 \%)$ & \\
\hline Adjunto $n=141$ & $51(36,2 \%)$ & 0,02 & $41(29 \%)$ & NS & $24(17 \%)$ & NS \\
\hline Residente $n=73$ & $15(20,5 \%)$ & & $18(24,6 \%)$ & & $11(15 \%)$ & \\
\hline Quiste roto $n=11$ & $4(36,7 \%)$ & NS & $5(45,4 \%)$ & NS & $3(27,3 \%)$ & NS \\
\hline Quiste íntegro $n=203$ & $62(30,5 \%)$ & & $55(27 \%)$ & & $33(16,2 \%)$ & \\
\hline Adherensiolisis $n=69$ & $32(46,4 \%)$ & 0,001 & $25(36,2 \%)$ & NS & $11(15,9 \%)$ & NS \\
\hline No adherensiolisis $n=145$ & $34(23,4 \%)$ & & $35(24,1 \%)$ & & $25(17,2 \%)$ & \\
\hline Implante peritoneal $n=39$ & $13(33,3 \%)$ & NS & $12(30,7 \%)$ & NS & $6(15,4 \%)$ & NS \\
\hline Sin implantes $n=175$ & $53(30,2 \%)$ & & $48(27,4 \%)$ & & $30(17,1 \%)$ & \\
\hline
\end{tabular}

NS: diferencia no significativa.

recurrencia ecográfica del endometrioma $(1,2,5)$ o sólo la reaparición de síntomas (1). Otros evalúan los síntomas y la ecografía $(5,6)$ y otros analizan el hallazgo ecográfico junto con el Ca 125 alto (7). En este estudio se han tomado como criterios todos los parámetros a considerar en el seguimiento de pacientes con endometriosis, evaluando el hallazgo ecográfico, la reaparición de síntomas y la nueva elevación del $\mathrm{Ca} 125$ en aquellos casos en los que estaba previamente elevado, intentado abarcar el problema en su totalidad.

Entre las pacientes que recidivaron, aparecieron casos con sólo reaparición de dismenorrea o sólo recidiva ecográfica. Cuando se dieron al menos dos de los criterios considerados, se aumentaron las posibilidades de atribuir el hallazgo a una recidiva endometriósica. Existe dificultad para asegurar que la dismenorrea o la imagen ecográfica sugestiva sean recidivas endometriósicas cuando aparecen aisladamente. 

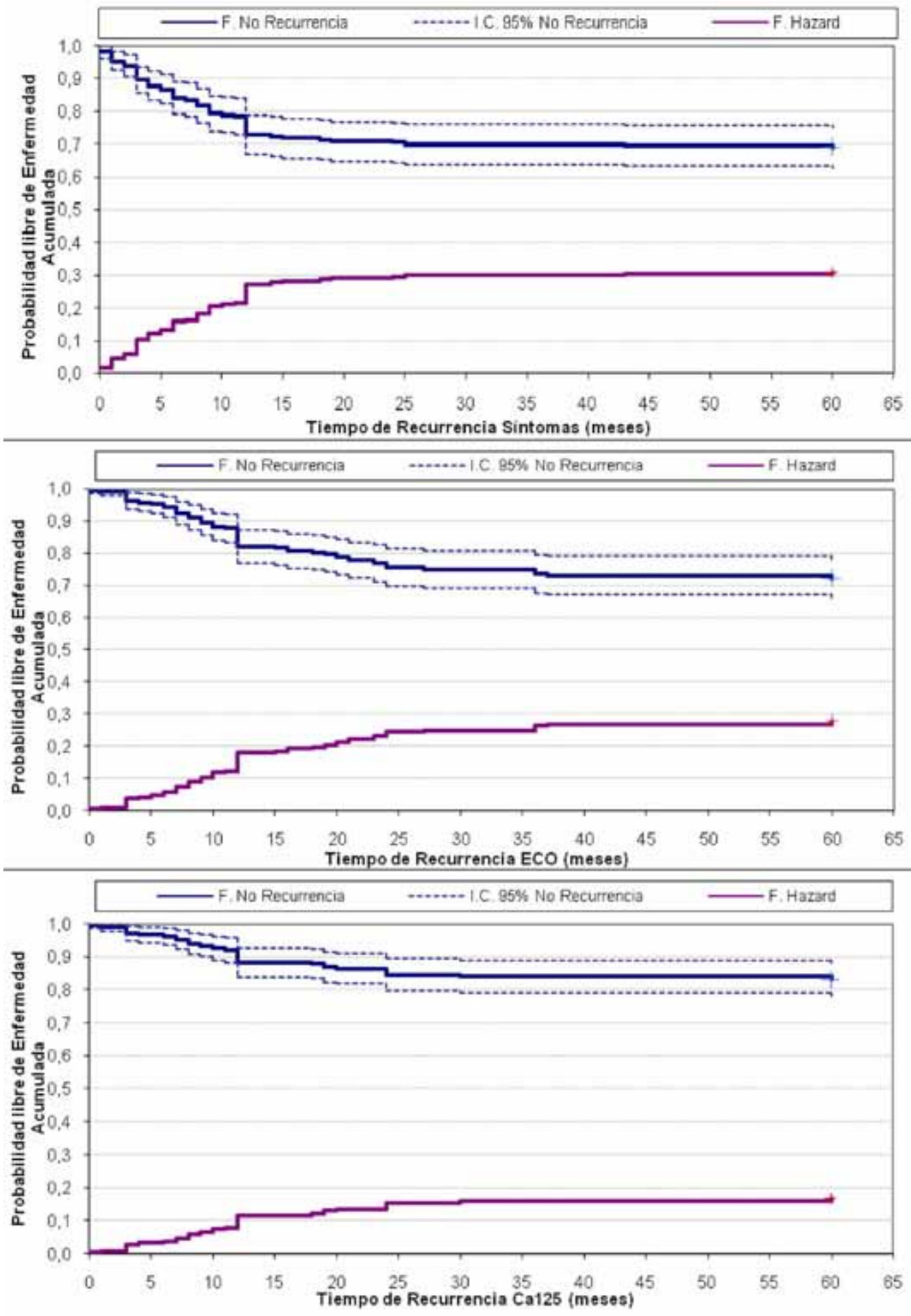

Figura 2. Curvas de Kaplan Meyer y de Hazard para evaluar tiempos de recurrencia sintomática, ecográfica y con nueva elevación de Ca 125. 
Lo más probable, en una paciente diagnosticada de endometriosis con muestra histológica, es que su nueva dismenorrea sea debida a esta enfermedad y que la imagen ecográfica sugestiva sea un endometrioma, pero no hay que obviar la existencia de dismenorrea esencial o el diagnóstico diferencial con otros quistes ováricos. Abbott y cols (4), informan que $1 / 3$ de las pacientes que se reoperaron por dolor posoperatorio no presentaron evidencia clínica laparoscópica, ni histológica, de endometriosis y persistió la dismenorrea.

Existe de igual modo un dilema clave a la hora de distinguir recurrencia de persistencia de la enfermedad. Hay escasos estudios que aborden este parámetro, y podría tratarse de dos modos de recurrencia. No está claro si la recurrencia ocurre in situ, por crecimiento de lesiones endometriósicas residuales, no detectables por laparoscopia o bien, ocurre de novo por el desarrollo de nuevas lesiones, o bien se trata de una combinación de ambas; se ha planteado como límite diferencial los 4-12 meses desde la cirugía (8). Pero no existe certeza de que a partir de ese tiempo sea una recurrencia y no una persistencia con un periodo de latencia y reactivación posterior.

La endometriosis se ha documentado tras 3-5 años de la menarquia, por lo que si las recurrencias se consideran de novo sería razonable que el periodo libre de recidiva fuese de al menos 36 meses y menor en la recurrencia in situ. Liu y cols (9), informan una tasa constante hazard en los 2830 meses tras la cirugía en los que la paciente no es vulnerable. A partir de ese periodo las tasas de dismenorrea y de endometrioma ecográfico incrementan dramáticamente, siendo más pronunciado en dismenorrea. Según esta premisa, los resultados expuestos en este estudio podrían estar más en relación con recurrencia in situ, observándose más recidivas en los primeros 20 meses.

Atendiendo a los resultados obtenidos, la existencia de dolor previo a la cirugía (dismenorrea, dispareunia) fue el factor más claramente asociado a la recidiva sintomática y ecográfica. La existencia de mayor dispareunia previa, también se asoció a mayor tasa de nueva elevación del $\mathrm{Ca} 125$. Este riesgo de recidiva sintomática y ecográfica apareció además asociado al grado de dismenorrea y dispareunia previas a la cirugía, de modo que las pacientes más sintomáticas son las que tienen más riesgo de recidiva futura. Así, en gran parte, la clínica inicial de la paciente será la que nos ponga en anticipo de la evolución posterior. La dismenorrea pos cirugía es la forma más común de presentación de recidiva, dato confirmado por otros estudios (4).
Centrándonos en la recurrencia de dismenorrea, la asociación con la edad joven ha sido evidenciada también por otros autores, que relacionaron la juventud a mayor recurrencia pero ecográfica a los 21-24 meses (9). Otros, sin embargo, no hayan relación entre edad y recidiva $(1,2)$.

La recidiva de dismenorrea se asoció también de forma relevante con la realización de adherensiolisis intraquirúrgica extensa. Las extensas adherencias, especialmente en anejos y fondo de saco de Douglas, son un factor asociado con la recurrencia de dismenorrea (1). Estos casos podrían enmarcarse en una enfermedad más agresiva y/o con mayor tendencia a re-formación de adherencias. También es posible que, escondidas entre las adherencias, queden pequeñas lesiones realizándose cirugía incompleta, o bien que persistan adherencias que pasen inadvertidas cuando son múltiples y extensas. Todo ello, obligaría a realizar una extirpación completa de las lesiones visibles y las adherencias, especialmente en aquellos casos en los que los síntomas sean la molestia principal.

El tratamiento médico pre y pos quirúrgico de los endometriomas ováricos es objeto de debate, pero existe acuerdo en afirmar que su eficacia como terapia exclusiva no ha sido demostrada. Conduciría a una reducción de volumen más que a una completa regresión del quiste. Así, el fallo del manejo médico sería debido a la persistencia de tejido endometriósico.

Previamente a la cirugía no hay indicaciones para prescribir terapia médica (8), y en cuanto a la administración posterior, existen datos dispares. La mayoría de trabajos afirman que no afecta significativamente a la recurrencia ni clínica ni ecográfica de endometriosis $(2,9,10)$. Pero algunos autores concluyen que los anticonceptivos orales previenen la recurrencia del endometrioma y otros encuentran cierto beneficio en el dolor con el tratamiento médico tras la cirugía (8). La terapia médica se prescribe tanto para intentar disminuir el riesgo de recurrencia como para aumentar el periodo libre de dolor. Es posible que permita disminuir el dolor a corto plazo, pero con el seguimiento más prolongado no presente un efecto protector para la recurrencia sintomática como describieron Muzzi y cols (10).

Los resultados obtenidos de mayor recurrencia sintomática a los 5 años entre las pacientes que recibieron tratamiento, podrían estar en relación bien con el hecho de la indicación del mismo, que se pauta a pacientes con síntomas severos, enfermedad avanzada y durante un corto periodo de tiempo, o bien con el hecho de persistencia de la enfermedad que permanecería latente durante el 
tratamiento reactivándose posteriormente. De cualquier modo, la endometriosis ovárica no debe ser considerada una contraindicación para el uso de anticonceptivos.

Analizando las características de la cirugía, se apreciaron más recidivas sintomáticas en pacientes a las que se realizó cirugía conservadora llevada a cabo por un médico adjunto. Con la anexectomía se abarcaría mayor cantidad de tejido permitiendo incluir en la pieza posibles pequeñas lesiones inadvertidas y facilitando la adherensiolisis en casos necesarios. Podría ser una opción válida en pacientes con mayor edad o sin deseo genésico. La quistectomía puede resultar más dificultosa en determinados casos aumentando la posibilidad de dejar tejido residual. Por otra parte, las menores tasas de reaparición del dolor en cirugías realizadas por residentes podrían estar en relación tanto al hecho de que el segundo cirujano fuese más experto como a la selección de casos menos dificultosos.

Dentro de los factores asociados a la recidiva ecográfica, además de la existencia y del grado de dismenorrea y dispareunia previas a la cirugía, se incluyó la infertilidad. La mayoría de las pacientes infértiles no recurren ecográficamente y la recurrencia ecográfica es más frecuente en no infértiles. La infertilidad parece estar asociada a cuadros más severos con importantes adherencias que produzcan oclusión tubárica y con pérdida de tejido ovárico. Sin embargo, si la cirugía es óptima la recurrencia ecográfica no tiene porqué aumentar frente a las pacientes fértiles.

La gestación posquirúrgica es considerada por diversos autores como un factor protector (2). Sin embargo, es en los primeros meses tras la intervención en los que podría ejercer su protección, atribuida a la supresión del crecimiento de la lesiones gracias al efecto de la progesterona (8). Los resultados expuestos son a los 5 años, evaluando la implicación a largo plazo de este factor y no mostrando beneficios en el seguimiento.

El Ca 125 se expresa principalmente en endometriosis avanzadas. Tiene su utilidad en el seguimiento para supervisar la respuesta al tratamiento, de tal forma que una elevación posquirúrgica persistente, predice un pronóstico relativamente desfavorable. De acuerdo con los resultados expuestos, predeciría mayor probabilidad de recurrencia ecográfica. Las pacientes con nueva elevación de Ca 125 tras la cirugía tuvieron cifras mayores del marcador y mayor dispareunia previas a la cirugía. La dispareunia está en relación con la extensión de adherencias pélvicas y como se ha mencionado el Ca 125 orienta a cuadros severos, siendo los resul- tados obtenidos coherentes con estos hechos.

El resto de factores estudiados no se relacionaron claramente con la recurrencia endometriósica. Si el tamaño del quiste no es un dato relevante en la recurrencia, en ausencia de síntomas marcados, habría que cuestionarse si habría que operarlos y con qué tamaño hacerlo. La necesidad de muestra histológica para descartar malignidad obligaría a llevar a cabo cirugía pero posiblemente en quistes $>4-5 \mathrm{~cm}$.

Centrándonos en las pacientes que recidivaron, la mayoría de los casos se controló con tratamiento médico, llegando a la necesidad de segunda cirugía en el $25,8 \%$, cifra similar a la hallada por otros autores (4).

\section{CONCLUSIONES}

La existencia de dolor previo a la cirugía (dismenorrea y dispareunia) fue el factor más claramente asociado a la recidiva, tanto sintomática como ecográfica, estando este riesgo de recidiva asociado al grado de dismenorrea y dispareunia previas a la cirugía. Las pacientes jóvenes, con quistectomía y adherensiolisis intraquirúrgica, y a las que se administró tratamiento médico posterior fueron las de mayor riesgo de recidiva de dismenorrea.

\section{BIBLIOGRAFÍA}

1. Porpora MG, Pallante D, Ferro AM, Crisafi B, Bellati F, Panici PB. Pain and ovarian endometrioma recurrence after laparoscopic treatment of endometriosis: a longterm prospective study. Fertil Steril 2010;93:716-21.

2. Koga K, Takemura Y, Osuya Y, Yoshino O, Hirota Y. Recurrence of ovarian endometrioma after laparoscopic excision. Human Reprod 2006;21:2171-4.

3. Jones KD, Sutton CJ. Laparoscopic management of ovarian endometriomas: a critical review of current practice. Curr Opin Obstet Gynecol 2000;12:309-15.

4. Abbott JA, Hawe J, Clayton RD, Garry R. The effects and effectiveness of laparoscopic excision of endometriosis: a prospective study with 2-5 year follow-up. Hum Reprod 2003;18:1922-7.

5. Muzii L, Bellati F, Bianchi A, Palaia I, Manci N, Zullo MA, Angioli R, Panici PB. Laparoscopic stripping of endometriomas: a randomized trial on different surgical techniques. Part II: pathological results. Hum Reprod 2005;20:1987-92.

6. Vercellini $P$, Somigliana E, Daguati R, Vigano $P$, Meroni F, Crosignani G. Postoperative oral contraceptive exposure and risk of endometrioma recurrence. Am J Obstet Gynecol 2007;198:504 e1-e5.

7. Mossa B, Ebano V, Tucci S, Rega C, Dolce E, Frega A, Marziani R. Laparoscopic surgery for the management of ovarian endometriomas. Med Sci Monit 2010;16:MT45-50.

8. Guo SW. Recurrence of endometriosis and its control. 
Hum Reprod Update 2009;15:441-61

9. Liu X, Yuan L, Shen F, Zhu Z, Jiang H, Guo SW. Patterns of and risk factors for recurrence in women with ovarian endometriomas. Obstet Gynecol 2007;109:1411-20.

10. Muzzii I, Marana R, Caruana P, Catalano GF, Mar- gutti F, Panici PB. Postoperative administration of monophasic combined oral contraceptives after laparoscopic treatment of ovarian endometriomas: a prospective, randomized trial. Am J Obstet Gynecol 2000;183:588-92. 